IAU Colloquium 164: Radio Emission from Galactic and Extragalactic Compact Sources

ASP Conference Series, Vol. 144, 1998

J. A. Zensus, G. B. Taylor, G J. M. Wrobel (eds.)

\title{
Rotation-Measure Mapping of 3C 119
}

\author{
C. Flatters \\ National Radio Astronomy Observatory, Socorro, NM, USA
}

Abstract. The compact steep-spectrum source $3 \mathrm{C} 119$ has been mapped using the VLBA at four frequencies spread over the $5 \mathrm{GHz}$ observing band. The high rotation-measure reported at short wavelengths is found to continue unbroken beyond the depolarization wavelength indicating that 3C 119 is depolarized by an external medium.

\section{Introduction}

3C 119 is a compact steep-spectrum source that has been identified with a quasar at $z=1.023$ (Eracleous \& Halpern 1994) and is known to have an unusually high rotation measure of $1728 \mathrm{rad} \mathrm{m}^{-2}$ and a depolarization wavelength between 3 and $6 \mathrm{~cm}$ (Kato et al. 1987). Previous VLBI observations (Fanti et al. 1986; Rendong et al. 1991) show that the radio structure of 3C 119 is extremely distorted. Ren-dong et al. suggest that this structure may result from a precessing jet viewed from inside the cone of precession or from the deflection of the jet by a dense, external medium.

\section{Observations}

3C 119 was observed with the VLBA on March 271995 using four IFs spread across the $5 \mathrm{GHz}$ VLBA observing band in order to see if there is any detectable rotation-measure structure. A rotation measure of $1728 \mathrm{rad} \mathrm{m}^{-2}$ would correspond to a total rotation of almost $50^{\circ}$ between the two most-widely separated IFs.

Each IF was mapped separately in polarization and the polarization position angles were calibrated with reference to observations of OJ 287 which was assumed to have a polarized position angle of $75^{\circ}$ (taken from the nearest observation of OJ 287 in the UMRAO database).

\section{Results}

The total intensity maps show three bright components and some indications of a jet joining them (Fig. 1, left). Fanti et al. (1986) show that Component D (using their nomenclature) has a flat spectrum and can be identified as the core while $\mathrm{A}$ and $\mathrm{B}$ are both steep spectrum components.

The polarized emission is dominated by Component $\mathrm{A}$, which is $2 \%$ polarized. The polarized structure is resolved (Fig. 1, right) with a brighter, extended region $(\mathrm{A} 0)$ and a dimmer, compact component (A1). The segment of the jet between Components $\mathrm{A}$ and $\mathrm{B}$ appears to be more closely aligned with $\mathrm{A} 1$ than A0.

Component $\mathrm{A} 0$ has a rotation measure of $1499 \pm 157 \mathrm{rad} \mathrm{m}^{-2}$ while A1 has a rotation measure of $1605 \pm 250 \mathrm{rad} \mathrm{m}^{-2}$, both of which are consistent with the Kato et al. value. 

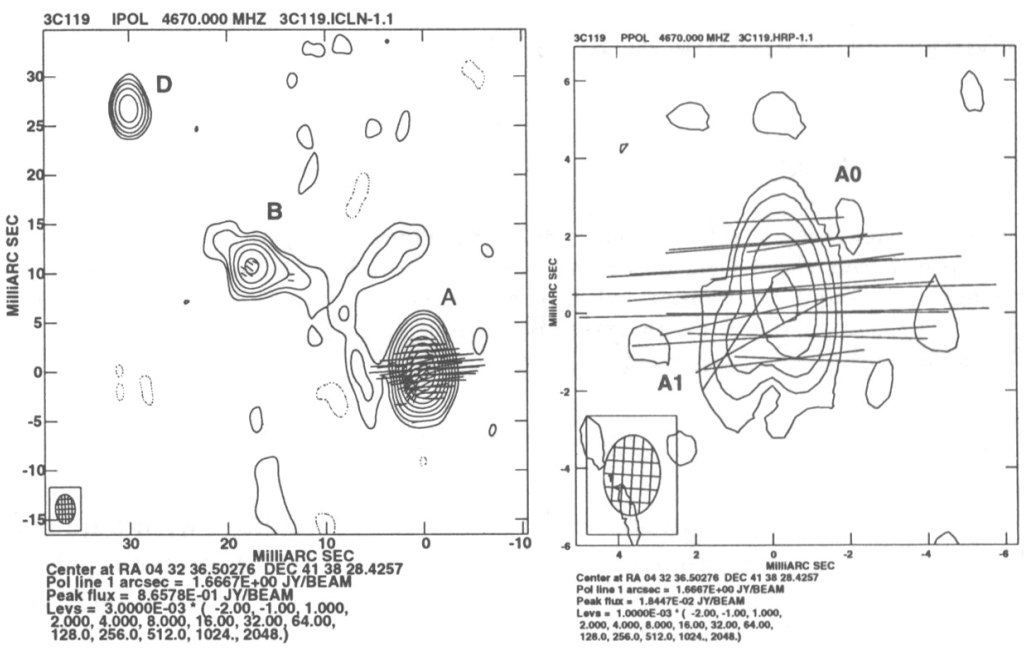

Figure 1. Electric polarization vectors overlayed on contours of total intensity using natural weights (left) and an expanded view of Component A with contours of polarized intensity using uniform weighting (right).

Polarization is also detected in Component $\mathrm{B}$ at the $1.2 \%$ level with a rotation measure of $779 \pm 597 \mathrm{rad} \mathrm{m}^{-2}$.

\section{Conclusions}

There is no sign of a departure from the $\lambda^{2}$ Faraday rotation law at the depolarization wavelength which is not consistent with models where the depolarization is due to thermal matter mixed with the emitting plasma (Burn 1966). This indicates that the depolarization must be caused by variations in the Faraday depth of material between the emitter and the Earth. The angular scale of these variations must be smaller than the beam ( 2 mas by 1 mas for uniform weighting). Such a small angular size suggests that the screen is more likely to be associated with the source than with our galaxy. This lends support to the hypothesis that $3 \mathrm{C} 119$ is interacting with a dense external medium.

Acknowledgments. The National Radio Astronomy Observatory is a facility of the National Science Foundation, operated under a cooperative agreement by Associated Universities, Inc. This research has made use of data from the University of Michigan Radio Astronomy Observatory which is supported by the National Science Foundation and by funds from the University of Michigan. Dave Graham originally pointed out that it should be possible to map rotation measures in 3C 119 using a single observing band.

\section{References}

Burn, B. J. 1966. MNRAS, 133,67-83.

Eracleous, M., \& Halpern, J. P. 1994. ApJS, 90, 1-30.

Fanti, C., et al. 1986. $A \& A, 170,10-19$.

Kato, T., et al. 1987. Nature, 329, 223-224.

Ren-dong, et al. 1991. $A \& A, 245,449-453$. 